\title{
Observation of Localized States in Lieb Photonic Lattices
}

\author{
Rodrigo A. Vicencio, ${ }^{1, *}$ Camilo Cantillano, ${ }^{1}$ Luis Morales-Inostroza, ${ }^{1}$ Bastián Real, ${ }^{1}$ Cristian Mejía-Cortés, ${ }^{1, \dagger}$ \\ Steffen Weimann, ${ }^{2}$ Alexander Szameit, ${ }^{2}$ and Mario I. Molina ${ }^{1}$ \\ ${ }^{1}$ Departamento de Física, MSI-Nucleus on Advanced Optics, and Center for Optics and Photonics (CEFOP), \\ Facultad de Ciencias, Universidad de Chile, Santiago 7800003, Chile \\ ${ }^{2}$ Institute of Applied Physics, Friedrich-Schiller-Universität Jena, Max-Wien-Platz 1, 07743 Jena, Germany
}

(Received 9 December 2014; published 15 June 2015)

\begin{abstract}
We present the first experimental demonstration of a new type of localized state in the continuum, namely, compacton-like linear states in flat-band lattices. To this end, we employ photonic Lieb lattices, which exhibit three tight-binding bands, with one being perfectly flat. Discrete predictions are confirmed by realistic continuous numerical simulations as well as by direct experiments. Our results could be of great importance for fundamental physics as well as for various applications where light needs to be conducted in a diffractionless and localized manner over long distances.
\end{abstract}

DOI: 10.1103/PhysRevLett.114.245503

PACS numbers: 42.82.Et, 78.67.Pt, 63.20.Pw

The localization of excitations in periodical lattices usually must rely on some form of defect, either pointlike or extended, linear or nonlinear, or simply disorder, in order to produce localized modes. However, there is another way of accomplishing this, in which the lattice remains perfectly periodic but is able to support localized states. In that case, one can find extremely localized entities that do not diffract at all, and that remain localized by virtue of a perfect geometric phase cancellation condition. This implies the possibility of using a judicious combination of these modes to transmit or localize information on specific regions of a lattice, without any distortion.

A Lieb lattice $[x-y$ pattern in Fig. $1(a)]$ is a square depleted lattice that is essentially a two-dimensional counterpart of the "perovskite" structure, which is ubiquitous in nature. The $\mathrm{CuO}_{2}$ planes of cuprate superconductors are perhaps the most famous example [1,2], but in fact many layered oxides coordinate in this fashion. Initial interest on this lattice began when ferromagnetism was found on the flat band at half filling [3]. Later, it was proven that ferromagnetism in this lattice was robust against spin wave excitations [4]. This lattice also displays unusual topological properties; for instance, in the presence of a uniform magnetic field, the flat band remains flat because of topological reasons [5]. The flat band touches two linearly dispersing intersecting bands at a single Dirac point. In the presence of Kerr nonlinearity the system may exhibit novel conical diffraction at the Dirac cone [6,7]. Very recently, the effect of considering correlated disorder on a Lieb lattice was studied [8], where a square-root singularity in the density of states was predicted. The Lieb lattice can now be realized by, e.g., manipulating cold atoms in optical lattices [9-11] and by direct laser writing of optical waveguides $[7,12,13]$.

The presence of a flat band in the spectrum of a Lieb lattice implies the existence of entirely degenerate states, the superposition of which displays no dynamical evolution. This allows the formation of four-site ring structures [see dashed line in Fig. 1(a)] that are completely localized in space, constituting a localized state in the continuum. The formation of those localized states arises from the high degeneracy of zero-frequency eigenmodes, as was recently shown in the context of chiral systems connected to a continuum [14]. In these systems, there exist zero-energy states where the wave function has finite amplitude in only one of the subsystems defined by the chiral symmetry. When the system is coupled to leads with a continuum energy band, some of these states remain bounded. These localized states are reminiscent of localized states in the continuum (BIC), originally predicted for nontranslationally invariant potentials [15]. However, the BIC concept has been expanded to different types of potentials, while retaining the principal property of being localized in space and existing inside the continuum, as recently investigated theoretically $[16,17]$ and experimentally $[18-21]$ in different photonics setups.

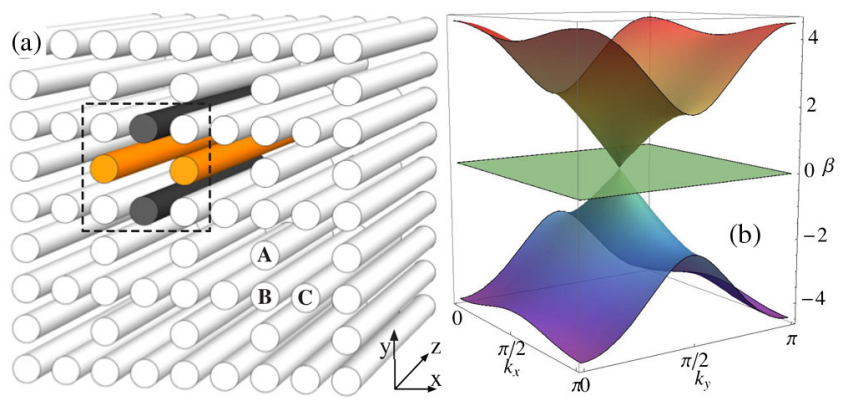

FIG. 1 (color online). (a) A Lieb photonic lattice with sites $A, B$, and $C$ defining the unitary cell. The dashed-line region encloses a four-site ring-mode profile, where different colors represent different amplitudes and different phases. (b) Linear spectrum of an anisotropic Lieb lattice for $V_{y}=2 V_{x}$. 
In this Letter, we demonstrate theoretically and experimentally the existence of new localized states consisting of compacton-like linear states residing in the linear band of a perfectly periodic Lieb lattice. They can be considered as a special type of BIC that can be located in any position of the lattice; this is a special property of flat-band states. This compacton-like localized entities do not diffract upon propagation, allowing in this manner the distortionless transmission of information along an optical channel [22].

The evolution of light along the $z$ direction on a Lieb photonic lattice, sketched in Fig. 1(a), composed by weakly coupled identical optical waveguides, is well described by a discrete linear Schrödinger equation [23,24],

$$
-i \frac{d u_{\vec{n}}}{d z}=\beta_{\vec{n}} u_{\vec{n}}+\sum_{\vec{m}} V_{\vec{n}, \vec{m}} u_{\vec{m}} .
$$

Here, $z$ is the coordinate along the propagation direction, and $u_{\vec{n}}$ corresponds to the light amplitude at the $\vec{n}$ th waveguide of a Lieb lattice. In our model, all waveguides are assumed to be identical; therefore, they possess the same propagation constants $\beta_{\vec{n}}=\beta_{0}$ [without loss of generality, we set $\beta_{0}=0$ in (1)]. The coupling (hopping) term between nearest-neighbor lattice sites $\vec{n}$ and $\vec{m}$ is denoted by $V_{\vec{n}, \vec{m}}$. The linear spectrum of a Lieb lattice is obtained by solving model (1) with a stationary ansatz, $u_{\vec{n}}(z)=u_{\vec{n}} \exp (i \beta z)$. We assume nearest-neighbor interactions only between sites $A, B$, and $C$ (unitary cell). To be more general, we consider an anisotropic lattice where the horizontal $\left(V_{x}\right)$ and vertical $\left(V_{y}\right)$ coupling coefficients can be different, but preserve the same lattice properties. For these three different lattice sites, we construct the coupling interactions considering a 2D Bloch wave vector $\vec{k}=$ $\left\{k_{x}, k_{y}\right\}$ and find three linear bands on this system [12],

$$
\beta(\vec{k})=0, \pm 2 \sqrt{V_{x}^{2} \cos ^{2}\left(k_{x}\right)+V_{y}^{2} \cos ^{2}\left(k_{y}\right)} .
$$

In Fig. 1(b), the linear spectrum (2) is shown inside the first Brillouin zone. Two bands are dispersive (nonzero curvature), possessing a particle-hole symmetry [25], where for each $\vec{k}$ there are two eigenfrequencies $\pm \beta(\vec{k})$. We observe that, for $V_{y}>V_{x}$, the dispersive bands show a different curvature depending on the direction of the $\vec{k}$ vector, where, in general, $y$-oriented waves propagate faster. These two bands are connected by a Dirac point at $\beta=0$. Exactly at this value, a completely flat (nondispersive) band is located. Diagonal interactions are considered negligible in our approach in order to preserve the flatness of this special band [6]. This assumption is well justified by the experiment.

In general, modes in the continuum of any periodic structure are completely extended. However, a flat-band system allows the formation of very localized, compactonlike states [26]. In a Lieb lattice, any closed ring (formed by eight sites) may support a ring mode, where $B$-site amplitudes are zero and the other two amplitudes satisfy the relation $V_{x} C=-V_{y} A$ for $k_{y}=k_{x}$, as sketched in Fig. 1(a). A measure of localization is provided by the participation ratio $R=\left(\sum_{\mathbf{n}}\left|u_{\mathbf{n}}\right|^{2}\right)^{2} / \sum_{\mathbf{n}}\left|u_{\mathbf{n}}\right|^{4}$, which takes the value 1 for a state localized at a single site and $N$ for a completely delocalized profile ( $N$ being the number of lattice sites). In our case, the ring modes have a participation ratio $R \leq 4$; i.e., they constitute a very compact localized state, appearing only by virtue of symmetry in a completely periodic lattice. This new type of BIC-like state can be placed at any position across the lattice and will propagate without diffraction along the longitudinal direction. Additionally, any linear combination of these states will be completely coherent and will propagate without any distortion, allowing for a highfidelity transmission of information along the longitudinal direction [22].

Tight-binding models, like model (1), are known to describe qualitatively well some particular systems that have short-range (weak coupling) interactions [23,24]. However, the description of a real lattice requires other methods to study the light dynamics. Our goal is to trace the optimal conditions for observing the described discrete phenomenology, avoiding extra interactions that could destroy the flatness of the band [6] and, therefore, impede the observation of linear compact states. In order to be closer to the conditions likely found in an experiment, we study dynamically a Lieb lattice using a continuous approach. We consider a linear paraxial wave equation [12] given by

$$
-i \frac{\partial}{\partial z} \psi(x, y, z)=\frac{\nabla_{\perp}^{2} \psi(x, y, z)}{2 k_{0} n_{0}}+k_{0} \Delta n(x, y) \psi(x, y, z),
$$

where $\psi$ is the envelope of the electric field, $k_{0}=2 \pi / \lambda$ is the wave number in free space, $\lambda$ is the wavelength, $n_{0}$ is the refractive index of the bulk material, and $\Delta n(x, y)$ corresponds to the refractive index structure that defines the Lieb photonic lattice. $\nabla_{\perp}^{2}=\partial_{x}^{2}+\partial_{y}^{2}$ corresponds to the transverse Laplacian operator. Once the geometry is fixed [in our case, waveguides are elliptical [12], implying a strong effective anisotropy: $V_{y} \sim 2 V_{x}$ in model (1)], we have essentially two free parameters left, the wavelength and the maximum index contrast $\delta n \equiv \max \left|\Delta n(x, y)-n_{0}\right|$. We implement a beam propagation method to solve Eq. (3) numerically and study the evolution of light across and along a Lieb lattice, considering a lattice period of $d=20 \mu \mathrm{m}$ and a propagation distance of $L=10 \mathrm{~cm}$. We consider green light of $\lambda=532 \mathrm{~nm}$ because it leads to a weaker coupling between lattice sites and, therefore, to a more discrete phenomenology.

By running our simulations at this wavelength, we obtain the results presented in Fig. 2. First, in Fig. 2(a), after exciting a $B$ site, we observe a small diffraction area, which 

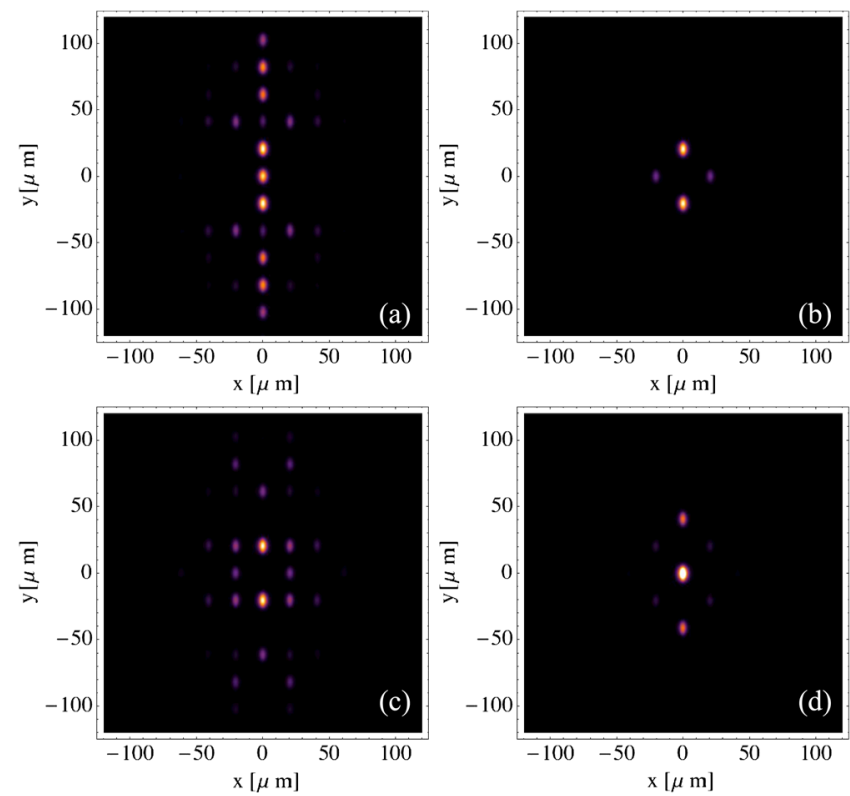

FIG. 2 (color online). Output profiles for different input conditions: (a) $B$-site excitation, (b) out-of-phase ring, (c) inphase ring, and (d) two added ring modes. $\lambda=532 \mathrm{~nm}$, $d=20 \mu \mathrm{m}, \delta n=0.7 \times 10^{-3}$, and $L=10 \mathrm{~cm}$. Color scheme: inverted black and white map.

is an indication of a weak-interaction (tight-binding) scenario. In addition, due to the waveguide ellipticity, we observe a very anisotropic and vertically oriented dispersion pattern, implying $V_{y}>V_{x}$ in model (1). In Fig. 2(b), we observe how a ring mode (i.e., four out-ofphase sites) could effectively be excited by choosing a right index contrast $\delta n$ (we ran several simulations to find that $\left.\delta n \gtrsim 0.67 \times 10^{-3}\right)$. In Fig. 2(c) we observe how a ring profile with uniform phase structure is destroyed and diffracts all over the lattice. This shows the importance of setting the right phase structure $(\pi)$ to effectively excite the flat-band states. Finally, as a proof of the numerical excitation of the ring mode, we generate a coherently combined state by summing two neighboring ring modes and propagating it along the system. As Fig. 2(d) shows, we observe the output pattern of this combined state, showing a perfect propagation of ring-mode combinations. We also generated different simple and complex linear combinations; all of them propagated without noticeable distortion.

The excitation of these localized states also gives rise to the onset of different excited eigenstates coming from the complete band structure of the lattice; in our numerical simulations, this manifests itself as a very weak (negligible) background radiation. We perform a longitudinal Fourier transform analysis [22] and study the excited frequency spectrum along the dynamics. We observe the presence of a large and thin peak in the region of the flat band, with a lower excitation of higher bands. By increasing the index contrast $\delta n$, we notice that this flatband peak increases and the gap between the nearest excited bands widens. Additionally, we perform simulations for propagation distances up to $L=50 \mathrm{~cm}$ to study the robustness of the ring-mode excitation. By inspecting the excited spectrum, we observe that the peak related to the flat band remains strongly excited in comparison to the rest of the excited spectrum. Although a realistic excitation of a ring mode is not perfect, as it is in a discrete nearest-neighbors model, we numerically find that the generated background radiation is very weak and can be considered negligible in comparison to the excited localized state. For distances $d$ and $L$ considered in this Letter, the effect of next-nearest neighbor interactions appears to be negligible as well. This is due to the weak coupling interaction coming from well-separated waveguides, which makes model (1) a valid approximation to describe the dynamics on this lattice.

To perform the experiments, we fabricate a Lieb photonic lattice using the direct femtosecond laser-writing technique on a $L=10 \mathrm{~cm}$-long fused silica glass wafer [27], as sketched in Fig. 3(a). In order to test the quality of this lattice, we launch white light at the input facet and take a microscope image at the output of the crystal [see Fig. 3(b)]. In this figure, we observe the propagating modes of each waveguide, which show a noticeable ellipticity that strongly affects the coupling interactions between nearest neighbors. We observe more evanescent light in between

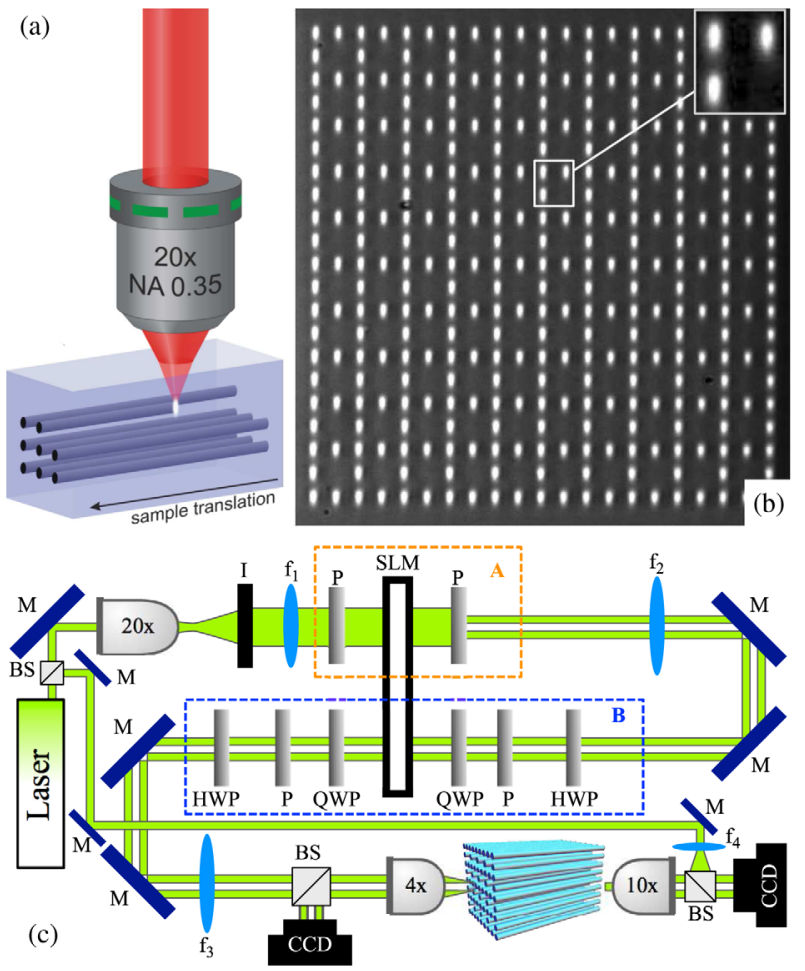

FIG. 3 (color online). (a) Femtosecond laser-writing technique. (b) Microscope image at the output facet of a Lieb lattice for white-light propagation. (c) Experimental setup to study the propagation of light patterns on a Lieb lattice. 
vertical sites than in between horizontal ones [see inset in Fig. 3(b)], due to the effective anisotropic coupling $\left(V_{y}>V_{x}\right)$.

The experimental observation of ring modes involves several challenging stages for the preparation of a proper initial condition. We essentially require the creation of the appropriate amplitude and phase pattern to be used as an input excitation, at the input facet of the array. In Fig. 3(c) we describe our experimental setup. We use a Holoeye LC2012 transmission spatial light modulator (SLM) to modulate, simultaneously, the amplitude and phase of a broad beam. We split the SLM display into two parts. In the first path (region $A$ ), we modulate the amplitude of the beam by tuning the angle of two polarizers $(P)$. We generate a pattern of several light disks of given radius (to be adjusted to match waveguides at the input facet) and given geometry (considering the Lieb structure). Once this modulation is achieved, we pass this light pattern through the second part of the SLM to modulate the amplitude profile in phase $(0$ or $\pi$ ). This modulation is performed in region $B$, by using an array of tuned half-wave (HWP) and quarter-wave (QWP) plates, and polarizers (all components were carefully tuned to optimize the required phase modulation, as well as the intensity of the profile and the respective input polarization [28]). After this stage, we obtain an amplitude- and phase-modulated light pattern, with a given polarization. In our experiment, light is polarized in the horizontal direction $\hat{x}$, in order to observe a larger diffraction area [29]. Finally, we inject this modulated pattern at the input facet of our Lieb photonic lattice by using a $4 \times$ microscope objective (MO), and observe the input profile with a CCD camera after being reflected on a beam splitter (BS). We obtain the output profile by using a $10 \times \mathrm{MO}$ and a CCD camera. To study the phase structure of different profiles, we interfere the input and the output patterns with a wide-tilted plane wave.

We start studying the bulk diffraction by using a $B$-site excitation, which is the input condition that better excites the dispersive part of the linear spectrum [12]. We inject light at the center of the lattice and observe the diffraction pattern shown in Fig. 4(a). We found an excellent agreement with respect to our numerical results [see Fig. 2(a)], which is very important for the calibration of the effective index contrast to be used in our simulations. Then, we prepare a symmetric ring-mode input profile, having four light disks with equal amplitude and a staggered phase structure, and observe its perfect propagation along the lattice [see Fig. 4(b)]. As the input profile is close but not the exact ring mode of the system, the light relaxes to this nonsymmetric configuration due to the anisotropic coupling coefficients. By increasing the image contrast (not shown here), we observe that a very weak radiation is also generated across the lattice, which is a manifestation of the presence of dispersive bands in the system. By interfering the input and output ring-mode profiles with a tilted plane
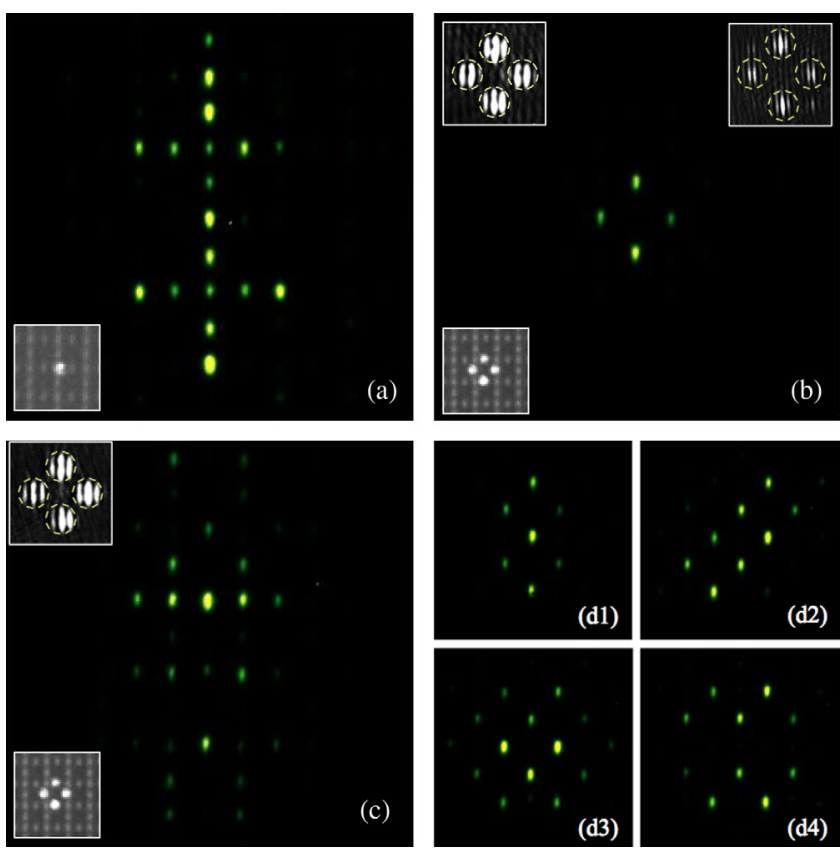

FIG. 4 (color online). Output experimental profiles for different input conditions: (a) $B$-site excitation, (b) out-of-phase ring, (c) in-phase ring, and (d) composed states excitation. In (a)-(c) lower-left insets show the input intensity profile. Upper insets in (b) and (c) show an interferogram of the ring mode with a tilted plane wave for input (left) and output (right) profiles.

wave, we observe that the phase structure is exactly the expected one [see Fig. 4(b), inset]: There is a $\pi$-phase shift difference between neighboring ring sites, as predicted by the tight-binding model. This shows very nicely that the initial discrete model prediction is valid in a realistic environment, and that the fundamental properties of the Lieb lattice are in fact observable in this experiment. We further test the relevance of the phase structure on the input condition by using an in-phase four-site ring excitation, as shown in Fig. 4(c). The output pattern shows a destruction of the initial localized profile due to the excitation of different linear bands (the output phase profile is not shown). This experiment agrees well with our simulations shown in Fig. 2(c) with a difference of the symmetry of the observed profile, which is essentially due to intrinsic fabrication anisotropies or from input conditions that are not perfectly aligned.

Finally, in order to probe the excitation of ring modes as stationary states and their potential use for transmitting optical information in narrow lattice regions, we combine them in different configurations. First, we confirm our numerics from Fig. 2(d), by preparing an initial condition composed by two vertically added ring modes and observing its perfect propagation in Fig. 4(d1). This shows experimentally the robustness of the application of the discrete analysis. This pattern has no discernible background radiation and possesses the same predicted structure for the combination of two anisotropic ring modes. 
Additionally, the vertical combination was designed thinking on the highest possible vertical diffraction that could be excited; nevertheless, we observe a perfect localized propagation. Finally, we test other linear combinations. For example, in Fig. 4(d2) we observe the propagation of two noninteracting ring modes along a diagonal. We also study the propagation of more complex patterns by linearly combining four ring modes. We construct and propagate a completely additive combination of four rings [Fig. 4(d3)], and a horizontally added and vertically subtracted one [Fig. 4(d4)]. By carefully selecting the phase and location of these modes, different linear patterns can be propagated to create a code based on these highly localized fundamental states.

In conclusion, we have experimentally observed the excitation of a new special type of localized state embedded into the continuum, consisting of a compact mode residing in the flat band of a Lieb photonic lattice. We have matched the right realistic conditions to observe this very fundamental weak-coupling mode and demonstrated the reality of flat-band systems. We have demonstrated the need to have a correct phase structure to be able to excite these localized entities. Additionally, we have combined these modes to create composite coherent and localized states, which propagate without diffraction across the lattice. This shows the possibility of creating different patterns and propagating them as a secure image transmission mechanism. Our results show a new way to propagate localized patterns by using the very fundamental properties of flatband systems that can be found and studied in a broad class of physical systems, ranging from solid state and magnetism to photonics. Our results provide a novel scheme of confining and controlling light. We foresee possible applications in, e.g., telecommunication and sensing [30].

The authors wish to thank A. Desyatnikov for valuable discussions. This work was supported in part by Fondef IDeA CA13I10244, Fondecyt Grants No. 1120123, No. 3140608, and No. 1151444, Programa ICM Grant No. RC-130001, Programa de Financiamiento Basal Grant No. FB0824, the Deutsche Forschungsgemeinschaft (Grant No. 462/6-1), and the German Ministry of Education and Research (Center for Innovation Competence program, Grant No. 03Z1HN31).

*rodrigov@uchile.cl

${ }^{\dagger}$ Present address: Departamento de Física, Universidad del Atlántico, Barranquilla, Colombia.

[1] V. J. Emery, Phys. Rev. Lett. 58, 2794 (1987).

[2] R. T. Scalettar, D. J. Scalapino, R. L. Sugar, and S. R. White, Phys. Rev. B 44, 770 (1991).

[3] E. H. Lieb, Phys. Rev. Lett. 62, 1201(1989).

[4] K. Kusakabe and H. Aoki, Phys. Rev. Lett. 72, 144 (1994).
[5] H. Aoki, M. Ando, and H. Matsumura, Phys. Rev. B 54, R17296(R) (1996).

[6] D. Leykam, O. Bahat-Treidel, and A.S. Desyatnikov, Phys. Rev. A 86, 031805(R) (2012).

[7] F. Diebel, D. Leykam, S. Kroesen, C. Denz, and A. S. Desyatnikov, in Advanced Photonics, OSA Technical Digest (Optical Society of America, Washington, DC, 2014), paper NW3A.1.

[8] J. D. Bodyfelt, D. Leykam, C. Danieli, X. Yu, and S. Flach, Phys. Rev. Lett. 113, 236403 (2014).

[9] R. Shen, L. B. Shao, B. Wang, and D. Y. Xing, Phys. Rev. B 81, 041410 (2010).

[10] N. Goldman, D. F. Urban, and D. Bercioux, Phys. Rev. A 83, 063601 (2011).

[11] V. Apaja, M. Hyrkas, and M. Manninen, Phys. Rev. A 82, 041402 (2010).

[12] D Guzmán-Silva, C. Mejía-Cortés, M. A. Bandres, M. C. Rechtsman, S. Weimann, S. Nolte, M. Segev, A. Szameit, and R. A. Vicencio, New J. Phys. 16, 063061 (2014).

[13] S. Mukherjee, A. Spracklen, D. Choudhury, N. Goldman, P. Öhberg, E. Andersson, and R. R. Thomson, arXiv:1412.6342 [Phys. Rev. Lett. (to be published)].

[14] J. Mur-Petit and R. A. Molina, Phys. Rev. B 90, 035434 (2014).

[15] J. von Neumann and E. Wigner, Phys. Z. 30, 465 (1929).

[16] E. N. Bulgakov and A. F. Sadreev, Phys. Rev. B 78, 075105 (2008).

[17] M. I. Molina, A. E. Miroshnichenko, and Y.S. Kivshar, Phys. Rev. Lett. 108, 070401 (2012).

[18] D. C. Marinica, A. G. Borisov, and S. V. Shabanov, Phys. Rev. Lett. 100, 183902 (2008).

[19] Y. Plotnik, O. Peleg, F. Dreisow, M. Heinrich, S. Nolte, A. Szameit, and M. Segev, Phys. Rev. Lett. 107, 183901 (2011).

[20] S. Weimann, Y. Xu, R. Keil, A. E. Miroshnichenko, A. Tünnermann, S. Nolte, A. A. Sukhorukov, A. Szameit, and Y. S. Kivshar, Phys. Rev. Lett. 111, 240403 (2013).

[21] G. Corrielli, G. Della Valle, A. Crespi, R. Osellame, and S. Longhi, Phys. Rev. Lett. 111, 220403 (2013).

[22] R. A. Vicencio and C. Mejía-Cortés, J. Opt. 16, 015706 (2014).

[23] S. Flach and A. Gorbach, Phys. Rep. 467, 1 (2008).

[24] F. Lederer, G. I. Stegeman, D. N. Christodoulides, G. Assanto, M. Segev, and Y. Silberberg, Phys. Rep. 463, 1 (2008).

[25] J. He, Y.-X. Zhu, Y.-J. Wu, L.-F. Liu, Y. Liang, and S.-P. Kou, Phys. Rev. B 87, 075126 (2013).

[26] D. L. Bergman, C. Wu, and L. Balents, Phys. Rev. B 78, 125104 (2008).

[27] A. Szameit and S. Nolte, J. Phys. B 43, 163001 (2010).

[28] I. Moreno, P. Velásquez, C. R. Fernández-Pousa, M. M. Sánchez-López, and F. Mateos, J. Appl. Phys. 94, 3697 (2003).

[29] S. Rojas-Rojas, L. Morales-Inostroza, U. Naether, G. B. Xavier, S. Nolte, A. Szameit, R. A. Vicencio, G. Lima, and A. Delgado, Phys. Rev. A 90, 063823 (2014).

[30] C. W. Hsu, B. Zhen, J. Lee, S.-L. Chua, S. G. Johnson, J. D. Joannopoulos, and M. Soljačić, Nature (London) 499, 188 (2013). 